\section{Chronic Nonbacterial Osteomyelitis in a Child with Previous Juvenile Dermatomyositis}

\section{To the Editor:}

Chronic nonbacterial osteomyelitis (CNO) is a sterile osteitis of unknown etiology. The diagnosis is based on characteristic clinical, laboratory, and imaging features ${ }^{1}$. Currently, $\mathrm{CNO}$ is considered an autoinflammatory disorder. However, the association of $\mathrm{CNO}$ with inflammatory bowel diseases $^{2}$, psoriasis ${ }^{3}$, and vasculitis suggests that both innate and adaptive immunity may contribute to pathogenesis.

A 3-year-old girl presented with proximal muscle weakness, Gottron papules, and periorbital heliotrope rash. Erythrocyte sedimentation rate (ESR) was $30 \mathrm{~mm} / \mathrm{h}$ (normal $0-12 \mathrm{~mm} / \mathrm{h}$ ); creatine kinase, aspartate aminotransferase, and lactate dehydrogenase were normal. Antinuclear autoantibody (ANA) titers were 1:320; antibodies against Jo-1, ribonuclear proteins, Smith, Ro, La, Scl-70, and double-stranded DNA were not detected. Muscle biopsy result was consistent with dermatomyositis. She was started on oral corticosteroids $1.5 \mathrm{mg} / \mathrm{kg} /$ day and methotrexate 0.5 $\mathrm{mg} / \mathrm{kg}$ weekly. After 9 months, muscle strength normalized and treatment was discontinued. Family history revealed that her brother and maternal grandmother had psoriasis and a paternal uncle was diagnosed with Crohn disease (Figure 1).

When she was 10 years old, she developed right distal femoral pain, swelling, and warmth and had elevated ESR $(17 \mathrm{~mm} / \mathrm{h})$. Cultures from blood and from the affected bone were normal. A radiograph of the right femur revealed a mottled lucency (Figure 2A). Despite 2 months of intravenous antibiotics, the bone pain persisted. Magnetic resonance imaging (MRI) of the right leg showed a small sequestrum; bone biopsy was in keeping with nonbacterial osteomyelitis. The persistence of bone symptoms for another year, together with the results of a second bone biopsy, confirmed CNO. In addition, recurrent attacks of Raynaud phenomenon of the hands and feet commenced at 11 years of age, without additional features suggestive for connective tissue diseases. At age 17 years, she still had bone pain, swelling, and warmth. ESR was $20 \mathrm{~mm} / \mathrm{h}$ and serum levels of muscle enzymes were normal. Femoral radiographs demonstrated unilateral small lucencies (Figure 2B); whole-body MRI confirmed the femoral lesion (Figure 2C). ANA titers were 1:160, extractable nuclear antibodies and anti-Scl-70 were negative. Whole exome sequencing did not reveal frameshift, stop gain or loss, or nonsyn-

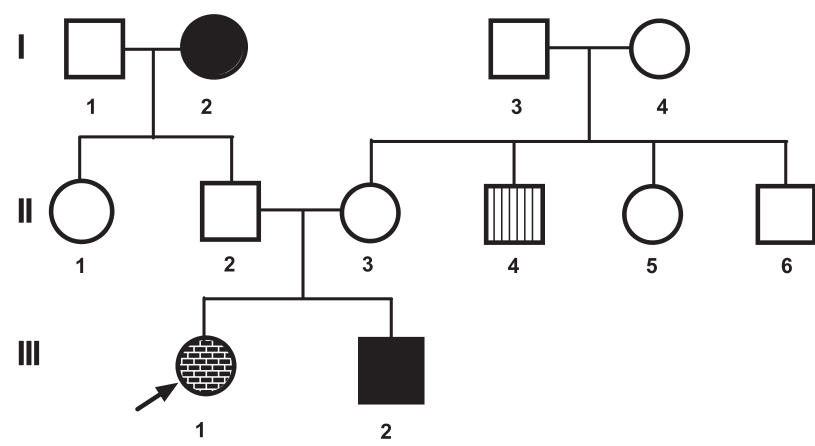

CNO, history of JDM

Psoriasis

Crohn disease

Figure 1. The family history of a child with chronic recurrent nonbacterial osteomyelitis and previous juvenile dermatomyositis (JDM) included psoriasis and Crohn disease. onymous single-nucleotide polymorphisms for lipin 2 (LPIN2) or proline-serine-threonine phosphatase interacting protein 2 (PSTPIP2). She was restarted on naproxen $15 \mathrm{mg} / \mathrm{kg} / \mathrm{day}$, with good response.

Several lines of evidence show that $\mathrm{CNO}$ and the multifocal type, chronic recurrent multifocal osteomyelitis (CRMO), are autoinflammatory diseases ${ }^{4}$. High-titer autoantibodies or autoreactive antigen-specific T cells are not found in CNO. Even though CD20-positive B cells and CD8-positive $\mathrm{T}$ cells are present at the site of bone inflammation, neutrophils, and in particular, macrophages are the immune cells that play an important role in $\mathrm{CNO}^{5}$.

In contrast, juvenile dermatomyositis (JDM) is a "mixed" disorder, with both autoimmune and autoinflammatory features. These patients exhibit a vast repertoire of autoantibodies and a strong $T$ cell response ${ }^{6}$. In addition, innate immunity also plays an important role in the pathogenesis of inflammatory myositis ${ }^{7}$. Complement activation induces secretion of proinflammatory cytokines and chemokines, facilitating the recruitment of macrophages, plasmacytoid dendritic cells (pDC), and T and B cells ${ }^{6}$. In $\mathrm{JDM}$, there is a strong type I interferon signature, originated from $\mathrm{pDC}$ and from the myocytes where the unfolded protein response (UPR) is activated. Moreover, activation of UPR may trigger the innate immune pathways ${ }^{7}$.

What do these diseases have in common? Both CNO and JDM are systemic immune-mediated inflammatory diseases, characterized by cytokine dysregulation. Tumor necrosis factor- $\alpha$, interleukin 10 (IL-10), and IL-1B play a role in pathogenesis of CRMO, whereas type I interferon has been implicated in $\mathrm{JDM}^{4,6,8,9}$. There is a strong innate response in the pathogenesis of both JDM and CNO. A link between these 2 diseases could be represented by IL-1ß, which can activate and amplify the intracellular T and $\mathrm{B}$ cell responses ${ }^{10}$.

We describe a unique case of CNO following JDM in a child with persistently positive ANA titers. From dysregulated adaptive immunity, characteristic for JDM, to prominent innate immune dysfunction, as is suspected in CNO, the child's immune system responded in distinctive ways to as-yet unknown stimuli. Specific stimuli could have driven the signaling through either immune pathway at different times during childhood. We speculate that the treatment of JDM could have partially reset the immune system in this patient. On a subclinical tolerogenic background, a second hit could have stimulated the rapid-acting, nonspecific innate immune system, leading to sterile bone inflammation. The sequential immune-mediated manifestations suggest there is crosstalk between the adaptive and innate immune arms, likely through common downstream signaling pathways and/or transcriptional targets. Autoinflammatory and autoimmune disorders are diseases of immune dysregulation, manifesting along a broad phenotypic spectrum.

DANIELA S. ARDELEAN, MD, FRCPC, Research Fellow, Division of Rheumatology, Department of Pediatrics, The Hospital for Sick Children, and Department of Immunology, University of Toronto, Toronto, Ontario; PETER B. DENT, MD, DCH, FRCPC, Professor Emeritus, Division of Rheumatology and Immunology, McMaster University, Hamilton, Ontario; POLLY J. FERGUSON, MD, FAAP, Associate Professor, Division of Pediatric Hematology, Oncology and Rheumatology, University of Iowa Children's Hospital, Iowa City, Iowa, USA; RONALD M. LAXER, MD, Professor, Division of Rheumatology, Department of Pediatrics, The Hospital for Sick Children, and Department of Medicine, University of Toronto, Toronto, Ontario, Canada.

Dr. Ardelean is supported by a Fellowship from CIHR/CAG/Abbott; and PGME/Department of Pediatrics, Department of Immunology, MOH/CIP, University of Toronto.

Address correspondence to Dr. R.M. Laxer, Division of Rheumatology, The Hospital for Sick Children, 555 University Avenue, Toronto, Ontario M5G 1X8, Canada.E-mail: ronald.laxer@sickkids.ca 

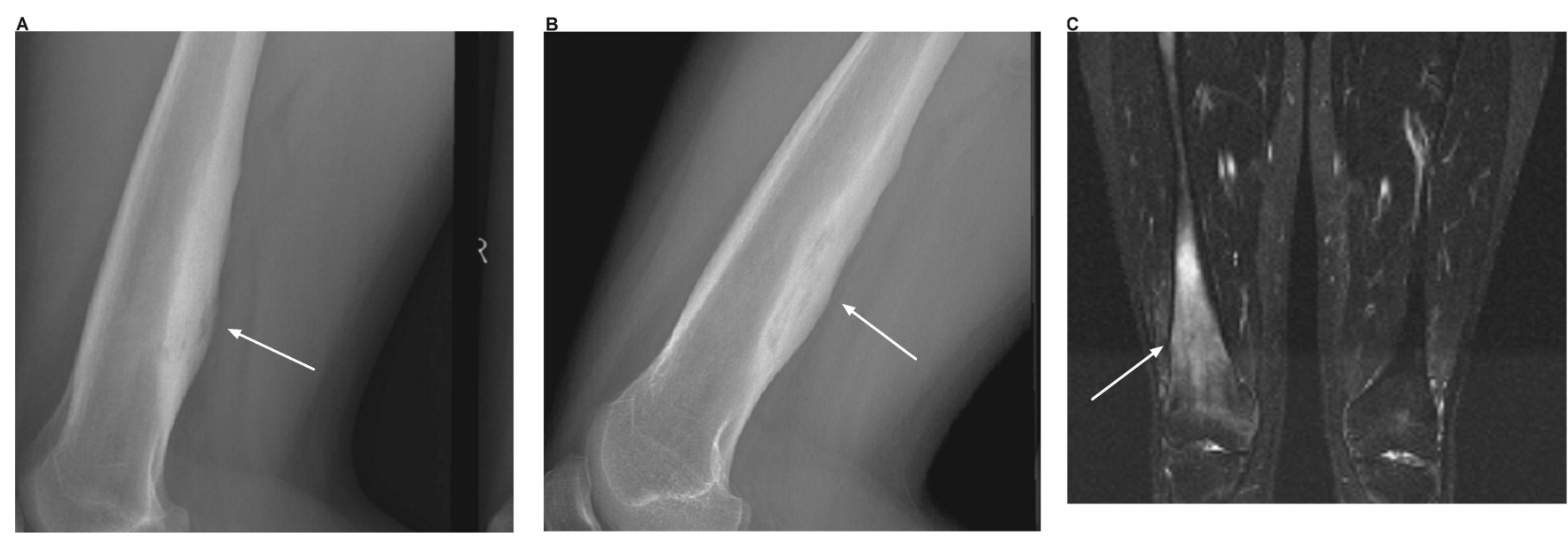

Figure 2. Images of chronic nonbacterial osteomyelitis of the femur in a child with previous juvenile dermatomyositis. Radiographs at (A) 10 and (B) 17 years of age showed posterior femoral lucencies and periosteal new bone formation. Coronal image of both femurs from total body magnetic resonance imaging (C) performed with inversion recovery sequences at 17 years of age. There is diffuse high signal of the distal right femur with associated low signal cortical thickening (courtesy of Dr. M. Ranson). White arrows show the affected bone area.

\section{REFERENCES}

1. Huber AM, Lam PY, Duffy CM, Yeung RS, Ditchfield M, Laxer D, et al. Chronic recurrent multifocal osteomyelitis: clinical outcomes after more than five years of follow-up. J Pediatrics 2002; 141:198-203.

2. Bousvaros A, Marcon M, Treem W, Waters $P$, Issenman R, Couper $\mathrm{R}$, et al. Chronic recurrent multifocal osteomyelitis associated with chronic inflammatory bowel disease in children. Dig Dis Sci 1999;44:2500-7.

3. Laxer RM, Shore AD, Manson D, King S, Silverman ED, Wilmot DM. Chronic recurrent multifocal osteomyelitis and psoriasis - A report of a new association and review of related disorders. Semin Arthritis Rheum 1988;17:260-70.

4. Ferguson PJ, El-Shanti HI. Autoinflammatory bone disorders. Curr Opin Rheumatol 2007;19:492-8.

5. Chitu V, Ferguson PJ, de Bruijn R, Schlueter AJ, Ochoa LA, Waldschmidt TJ, et al. Primed innate immunity leads to autoinflammatory disease in PSTPIP2-deficient cmo mice. Blood 2009;114:2497-505.
6. Robinson AB, Reed AM. Clinical features, pathogenesis and treatment of juvenile and adult dermatomyositis. Nat Rev Rheumatol 2011;7:664-75.

7. Park H, Bourla AB, Kastner DL, Colbert RA, Siegel RM. Lighting the fires within: The cell biology of autoinflammatory diseases. Nat Rev Immunol 2012;12:570-80.

8. Hamel J, Paul D, Gahr M, Hedrich CM. Pilot study: Possible association of IL10 promoter polymorphisms with CRMO. Rheumatol Int 2012;32:555-6.

9. Deutschmann A, Mache CJ, Bodo K, Zebedin D, Ring E. Successful treatment of chronic recurrent multifocal osteomyelitis with tumor necrosis factor-alpha blockage. Pediatrics 2005;116:1231-3.

10. Doria A, Zen M, Bettio S, Gatto M, Bassi N, Nalotto L, et al. Autoinflammation and autoimmunity: Bridging the divide. Autoimmun Rev 2012;12:22-30.

J Rheumatol 2013;40:3; doi:10.3899/jrheum.120963 